\title{
ON THE CLASS $\mathbb{A}_{1, \aleph_{0}}$
}

\author{
BEBE PRUNARU
}

(Communicated by John B. Conway)

\begin{abstract}
The solvability of certain systems of simultaneous equations in the predual of a dual operator algebra is studied. The main result is a geometric criterion for membership in the class $A_{1, \aleph_{0}}$.
\end{abstract}

Let $\mathscr{H}$ denote a separable, infinite-dimensional complex Hilbert space, and let $L(\mathscr{H})$ denote the algebra of all bounded linear operators on $\mathscr{H}$. A dual algebra is by definition a weak* closed unital subalgebra of $L(\mathscr{H})$; [1] contains an excellent account of the theory of dual algebras and a comprehensive bibliography up to 1985 .

In this paper we are concerned with several classes of contractions appearing in the theory of dual algebras. To be more explicit, recall that if $\mathscr{A}$ is a dual algebra and $m, n$ are cardinal numbers, $1 \leq m, n \leq \kappa_{0}$, then $\mathscr{A}$ is said to have property $\left(A_{m, n}\right)$ if each system of simultaneous equations

$$
\left[L_{i j}\right]=\left[x_{i} \otimes y_{j}\right], \quad 0 \leq i<m, 0 \leq j<n
$$

in the predual $Q_{\mathscr{A}}$ of $\mathscr{A}$ has a solution $\left\{x_{i} ; 0 \leq i<m\right\},\left\{y_{j} ; 0 \leq j<n\right\}$, where $x_{i}$ and $y_{j}$ are vectors from $\mathscr{H}$. (Here $[x \otimes y]$ denotes the class in $Q_{A}$ of the rank-one operator defined by $(x \otimes y)(z)=(z, y) x, z \in \mathscr{H}$.)

If $\rho>0$ then $\mathscr{A}$ has property $\left(A_{m, n}(\rho)\right)$ if for each $s>\rho$, vectors $x_{i}$ and $y_{j}$ can be chosen to satisfy the above equations and, moreover,

$$
\left\|x_{i}\right\|<\left(s \sum_{0 \leq j<n}\left\|\left[L_{i j}\right]\right\|\right)^{1 / 2}, \quad 0 \leq i<m,
$$

and

$$
\left\|y_{j}\right\|<\left(s \sum_{0 \leq i<m}\left\|\left[L_{i j}\right]\right\|\right)^{1 / 2}, \quad 0 \leq j<n .
$$

If $T \in L(\mathscr{H})$, then $\mathscr{A}_{T}$ denotes the dual algebra generated by $T$ in $L(\mathscr{H})$ and $Q_{T}$ denotes the predual $Q_{\mathscr{A}_{T}}$ of $\mathscr{A}_{T}$. As usual, $\mathbb{A}=\mathbb{A}(\mathscr{H})$ denotes the

Received by the editors March 21, 1988 and, in revised form, May 17, 1990.

1980 Mathematics Subject Classification (1985 Revision). Primary 47A15; Secondary 47A20.

Key words and phrases. Dual operator algebras. 
class of all absolutely continuous contractions (i.e., those for which the unitary summand is absolutely continuous) for which the Sz.-Nagy-Foias functional calculus is an isometry. If $m$ and $n$ are cardinal numbers, $1 \leq m, n \leq \aleph_{0}$, then

$\mathbb{A}_{m, n}=\left\{T \in \mathbb{A} ; \mathscr{A}_{T}\right.$ has property $\left.\left(A_{m, n}\right)\right\}$, and similarly for $\mathbb{A}_{m, n}(\rho)$.

If $m=n$, then one usually sets $\mathbb{A}_{n}=\mathbb{A}_{n, n}$ and $\mathbb{A}_{n}(\rho)=\mathbb{A}_{n, n}(\rho)$. In [3], B. Chevreau and C. Pearcy have given a sufficient condition for membership in the class $\mathbb{A}_{1}(\rho)$ [3, Theorem 4.4].

The purpose of this paper is to show that this condition is sufficiently strong to ensure the membership in the class $\mathbb{A}_{1, \aleph_{0}}(\rho)$. Before giving the main result, we recall some ideas introduced in [3].

Let $\mathscr{A} \subset L(\mathscr{H})$ be a dual algebra, and let $\theta \in[0,1)$. Then $E_{\theta}^{r}(A)$ denotes the set of all those $[L]$ in $Q_{\nsim}$ such that there exist sequences $\left\{x_{n}\right\}$ and $\left\{y_{n}\right\}$ in the unit ball of $\mathscr{H}$ satisfying

$$
\begin{aligned}
\lim _{n} \sup \left\|[L]-\left[x_{n} \otimes y_{n}\right]\right\| & \leq \theta, \\
\lim \left\|\left[x_{n} \otimes z\right]\right\|=0, & z \in \mathscr{H},
\end{aligned}
$$

and

$$
\left\{y_{n}\right\} \text { converges weakly to zero. }
$$

A set $E_{\theta}^{l}(\mathscr{A})$ can also be defined by replacing conditions (b) and (c) above with

$$
\lim _{n}\left\|\left[z \otimes y_{n}\right]\right\|=0, \quad z \in \mathscr{H},
$$

and

$$
\left\{x_{n}\right\} \text { converges weakly to zero. }
$$

If $0 \leq \theta<\gamma$, then a dual algebra $\mathscr{A} \subset L(\mathscr{H})$ is said to have property $E_{\theta, \gamma}^{r}\left(\right.$ respectively $\left.E_{\theta, \gamma}^{l}\right)$ if the closed absolutely convex hull of the set $E_{\theta}^{r}(\mathscr{A})$ (respectively $E_{\theta}^{l}(A)$ ) contains the closed ball $B_{\gamma}=\left\{[L] \in Q_{\mathscr{A}} ;\|[L]\| \leq \gamma\right\}$.

Suppose now that $T \in \mathbb{A}(\mathscr{H})$ and $B \in L(\mathscr{K})$ is its minimal coisometric extension. Then $B \in \mathbb{A}(\mathscr{K})$ and $B=S^{*} \oplus R$, where $S \in L(\mathscr{P})$ is a unilateral shift and $R \in L(\mathscr{R})$ is an absolutely continuous unitary operator.

Let $Q$ and $A$ denote the orthogonal projections of $\mathscr{K}$ onto $\mathscr{P}$ and $\mathscr{R}$, respectively. If $\mathscr{R} \neq\{0\}$, then one knows (cf. [3, Proposition 3.10]) that there exist a Borel subset $\sigma$ of the unit circle $\mathbb{T}$ and a subspace $\mathscr{R}_{0} \subset \mathscr{R}$, reducing for $R$ such that

( $\alpha) \quad m_{\mid \sigma}$ is a scalar spectral measure for $R$ (here $m$ denotes the Lebesgue measure on $\mathbb{T}$ );

(ß) $R_{0}=R_{\mid \mathscr{R}_{0}}$ is unitarily equivalent with the multiplication operator $M_{e^{i t}}$ on $L^{2}(\sigma)$; and

(y) $\overline{A \mathscr{H}}$ contains the subspace $\mathscr{R}_{0}^{+}$of $\mathscr{R}_{0}$ corresponding to the closure of polynomials in $L^{2}(\sigma)$. 
For any $T \in \mathbb{A}(\mathscr{H})$, let $\varphi: Q_{T} \rightarrow Q_{B}$ denote the canonical isometry defined by

$$
\langle f(T),[L]\rangle=\langle f(B), \varphi([L])\rangle, \quad f \in H^{\infty},[L] \in Q_{T} .
$$

One easily sees that

$$
\varphi\left([x \otimes y]_{T}\right)=[x \otimes y]_{B}, \quad x, y \in \mathscr{H},
$$

and

$$
[x \otimes z]_{B}=\left[x \otimes P_{H} z\right]_{B}, \quad x \in \mathscr{H}, \quad z \in \mathscr{K} .
$$

The main result of this paper is the following:

Theorem 1. Suppose $T \in \mathbb{A}(\mathscr{H})$ and $\mathscr{A}_{T}$ has property $E_{\theta, \gamma}^{r}$ for some $0<\theta<$ $\gamma$. Then, for each sequence of elements $\left\{\left[L_{j}\right] ; j \geq 1\right\}$ from $Q_{\mathscr{S}_{T}}$ such that $\sum_{j \geq 1}\left\|\left[L_{j}\right]\right\|^{1 / 2}<\infty$, there are vectors $x$ and $\left\{y_{j} ; j \geq 1\right\}$ in $\mathscr{H}$ such that

$$
\begin{aligned}
{\left[L_{j}\right] } & =[x \otimes y]_{j}, \quad j \geq 1, \\
\|x\| & \leq \frac{3}{1-(\theta / \gamma)^{1 / 2}} \sum_{j \geq 1}\left\|\left[L_{j}\right]\right\|^{1 / 2}, \\
\left\|y_{j}\right\| & \leq \frac{3}{1-(\theta / \gamma)^{1 / 2}}\left\|\left[L_{j}\right]\right\|^{1 / 2}, \quad j \geq 1 .
\end{aligned}
$$

In particular, $\mathscr{A}_{T}$ has property $\left(A_{1}, \widehat{\aleph}_{0}\right)$.

After this paper was completed, the author learned that B. Chevreau, G. Exner, and C. Pearcy have also proved Theorem 1, seemingly with different methods. Moreover, they showed that all c.n.u. contractions $T$ such that $A_{T}$ has property $E_{\theta, \gamma}^{r}$ are reflexive. Their results were announced in [2].

Let $A_{0}$ denote the orthogonal projection of $\mathscr{K}$ onto $\mathscr{R}_{0}$, and let $z \rightarrow\{z\}$ denote the isomorphism from $\mathscr{R}_{0}$ onto $L^{2}(\sigma(R))$. The following lemma is proved in [3]:

Lemma 1 [3, Theorem 3.11]. Suppose $T \in \mathbb{A}(\mathscr{H})$ and, for some $0<\theta<\gamma, \mathscr{A}_{T}$ has property $E_{\theta, \gamma}^{r}$. Suppose also that $[L] \in Q_{B}, 0<\rho<1, \varepsilon>0, \delta>0$, $a \in \mathscr{H}, w \in \mathscr{P}, b \in \mathscr{R}_{0},\left\{d_{s}\right\}_{s=1}^{t} \subset \mathscr{K}$, and $\left\{z_{l}\right\}_{l=1}^{r} \subset \mathscr{P}$ are given, so that

$$
\left\|[L]_{B}-[a \otimes(w+b)]_{B}\right\|<\delta .
$$

Then there exist $a^{\prime} \in \mathscr{H}, u \in \mathscr{H}, w^{\prime} \in \mathscr{P}, b^{\prime} \in \mathscr{R}_{0}$ such that the following 
are true:

$$
\begin{aligned}
\left\|[L]_{B}-\left[a^{\prime} \otimes\left(w^{\prime}+b^{\prime}\right)\right]_{B}\right\| & <\frac{\theta}{\gamma} \delta, \\
\left\|a^{\prime}-a\right\| & <3\left(\frac{\delta}{\gamma}\right)^{1 / 2}, \\
\left\|w^{\prime}-w\right\| & <\left(\frac{\delta}{\gamma}\right)^{1 / 2}, \\
\left\|b^{\prime}\right\| & <\frac{1}{\rho}\left\{\|b\|+\left(\frac{\delta}{\gamma}\right)^{1 / 2}\right\}, \\
\left|\left\{A_{0} a^{\prime}\right\}\left(e^{i t}\right)\right| & \geq \rho\left|\left\{A_{0}(a+u)\right\}\left(e^{i t}\right)\right|, \quad e^{i t} \in \mathbb{T} \\
\left\|\left[u \otimes d_{s}\right]\right\| & <\varepsilon, \quad 1 \leq s \leq t, \\
\left\|\left[\left(a^{\prime}-a\right) \otimes z_{l}\right]\right\| & <\varepsilon, \quad 1 \leq l \leq r .
\end{aligned}
$$

We are now prepared to prove the main result. Its proof follows the main ideas from [4, Lemma 5] and [3, Theorem 4.7].

Proof of Theorem 1. Let $\left\{\left[L_{j}\right]\right\}_{j=1}^{\infty} \subset Q_{T}$, and also let $\left[L_{j}^{\prime}\right]_{B}=\varphi\left(\left[L_{j}\right]_{T}\right)$ for $j \geq 1$.

Let $\delta_{j}>0$ such that $\sum \delta_{j}^{1 / 2}$ is finite. Without loss of generality, we may assume that $\left\|\left[L_{j}^{\prime}\right]\right\|<\delta_{j}$ for each $j$.

Let us set $\varepsilon_{j k}=\delta_{j}\left(\frac{\theta}{\gamma}\right)^{k}$ for all $j \geq 1$ and $k \geq 0$.

Let $\left\{s_{n}\right\}$ be a sequence of positive numbers strictly decreasing to $\frac{1}{2}$ such that $s_{1}=1$, and let $\rho_{n}=s_{n+1} / s_{n}, n \geq 1$.

Let $B: N \times N \rightarrow N$ be a bijection such that $j \leq j^{\prime}$ and $k \leq k^{\prime}$ implies

$$
B(j, k) \leq B\left(j^{\prime}, k^{\prime}\right) .
$$

Let $w_{j, 0}=0$ in $\mathscr{P}$ and $b_{j, 0}^{n}=0$ in $\mathscr{R}_{0}$, for all $j \geq 1$ and all $n \geq 1$.

We shall construct, by induction (on the range of $B$ ), sequences $\left\{x_{n}\right\} \subset \mathscr{H}$, $\left\{w_{j, k}\right\}_{j, k \geq 1}$ in $\mathscr{P}$ and, for $n \geq 1$, finite sequences $\left\{b_{j, k}^{n}\right\}_{B(j, k) \leq n}$ in $\mathscr{R}_{0}$ such that

(1) $\left[L_{j}^{\prime}\right]_{B}-\left[x_{n} \otimes\left(w_{j, k}+b_{j}^{n}, \hat{k}\right)\right]_{B} \|<\varepsilon_{j, k}, B(j, k) \leq n$;

(2) $\left\|x_{n}-x_{n-1}\right\|<3 \varepsilon_{j, k-1}^{1 / 2}$, for $n=B(j, k)$;

(3) $\left\|w_{j, k}-w_{j, k-1}\right\|<\varepsilon_{j, k-1}^{1 / 2}, \forall j, k \geq 1$;

(4) $\left\|b_{j, k}^{k}\right\|<\frac{1}{\rho_{n}}\left\|b_{j, k}^{n-1}\right\|$, if $n>B(j, k)$; and

(5) $\left\|b_{j, k}^{n}\right\|<\frac{1}{\rho_{n}}\left\{\left\|b_{j, k-1}^{n-1}\right\|+\varepsilon_{j, k-1}^{1 / 2}\right\}$ if $n=B(j, k)$.

For $n=1=B(1,1)$, we apply Lemma 1 , with $L=\left[L_{1}^{\prime}\right], \delta=\delta_{1}, \rho=\rho_{1}$, $a=0, b=0, w=0, d=0, z=0$ to find $x_{1} \in \mathscr{H}, w_{11} \in \mathscr{P}, b_{11}^{1} \in \mathscr{R}_{0}$ so 
that

$$
\begin{aligned}
\left\|\left[L_{1}^{\prime}\right]-\left[x_{1} \otimes\left(w_{11}+b_{11}\right)\right]\right\| & <\varepsilon_{11}, \\
\left\|x_{1}\right\| & <3 \delta_{1}^{1 / 2}, \\
\left\|w_{11}\right\| & <\delta_{1}^{1 / 2}
\end{aligned}
$$

and

$$
\left\|b_{11}^{1}\right\|<\frac{1}{\rho_{1}} \delta_{1}^{1 / 2}
$$

Suppose now that vectors $\left\{x_{1}, \ldots, x_{n}\right\}$ in $\mathscr{H},\left\{w_{j, k}\right\}_{B(j, k) \leq n}$ in $\mathscr{P}$, and $\left\{b_{j k}^{n}\right\}_{B(j, k) \leq n}$ in $\mathscr{R}_{0}$ have been chosen so that (1)-(5) are satisfied. Let $n+1=$ $B(p, q)$. Apply Lemma 1 with $[L]=\left[L_{p}^{\prime}\right], a=x_{n}, w=w_{p, q-1}, b=b_{p, q-1}^{n}$, $\rho=\rho_{n+1}, \delta=\varepsilon_{p, q-1}$,

$$
\begin{aligned}
& \left\{d_{s}\right\}=\left\{b_{j k}^{n}\right\}_{B(j, k) \leq n}, \\
& \left\{z_{l}\right\}=\left\{w_{j, k}\right\}_{B(j, k) \leq n},
\end{aligned}
$$

and $\varepsilon>0$ sufficiently small (to be determined later) to obtain $x_{n+1} \in \mathscr{H}$, $w_{p, q} \in \mathscr{P}, b_{p, q}^{n+1} \in \mathscr{R}_{0}$, and $u_{n+1} \in \mathscr{H}$ such that

$$
\begin{aligned}
\left\|\left[L_{p}^{\prime}\right]_{B}-\left[x_{n+1} \otimes\left(w_{p, q}+b_{p, q}^{n+1}\right)\right]_{B}\right\| & <\varepsilon_{p, q}, \\
\left\|x_{n+1}-x_{n}\right\| & <3 \varepsilon_{p, q-1}^{1 / 2}, \\
\left\|w_{p, q}-w_{p, q-1}\right\| & <\varepsilon_{p, q-1}^{1 / 2}, \\
\left\|b_{p, q}^{n+1}\right\| & <\frac{1}{\rho_{n+1}}\left\{\left\|b_{p, q-1}^{n}\right\|+\varepsilon_{p, q-1}^{1 / 2}\right\}, \\
\left|\left\{A_{0} x_{n+1}\right\}\left(e^{i t}\right)\right| & >\rho_{n+1}\left|\left\{A_{0}\left(x_{n}+u_{n+1}\right)\left(e^{i t}\right)\right\}\right|, \quad e^{i t} \in \mathbb{T}, \\
\left\|\left[\left(x_{n+1}-x_{n}\right) \otimes w_{j k}\right]\right\| & <\varepsilon \text { for } B(j, k) \leq n,
\end{aligned}
$$

and

$$
\left\|\left[u_{n+1} \otimes b_{j, k}^{n}\right]\right\|<\varepsilon \text { for } B(j, k) \leq n .
$$

Let us define for each $(j, k)$, with $B(j, k) \leq n$

$$
\left\{\overline{b_{j, k}^{n+1}}\right\}\left(e^{i t}\right)=\frac{\left\{A_{0}\left(x_{n}+u_{n+1}\right)\right\}\left(e^{i t}\right)}{\left\{A_{0}\left(x_{n+1}\right)\right\}\left(e^{i t}\right)} \cdot b_{j, k}^{n}\left(e^{i t}\right) \text { if }\left\{A_{0}\left(x_{n+1}\right)\left(e^{i t}\right)\right\} \neq 0
$$

and

$$
\left\{\overline{b_{j, k}^{n+1}}\right\}\left(e^{i t}\right)=0 \text { if }\left\{A_{0}\left(x_{n+1}\right)\left(e^{i t}\right)\right\}=0 .
$$

It follows that

$$
\begin{aligned}
b_{j, k}^{n+1} & \in \mathscr{R}_{0}, \\
\left\|b_{j, k}^{n+1}\right\| & <\frac{1}{\rho_{n+1}}\left\|b_{j, k}^{n}\right\|,
\end{aligned}
$$


and

$$
\left[x_{n+1} \otimes b_{j, k}^{n+1}\right]_{B}=\left[\left(x_{n}+u_{n+1}\right) \otimes b_{j, k}^{n}\right]_{B}
$$

for all $(j, k)$ such that

$$
B(j, k) \leq n .
$$

For $\varepsilon>0$ sufficiently small, we have

$$
\left\|\left[L_{j}^{\prime}\right]_{B}-\left[x_{n+1} \otimes\left(w_{j k}+b_{j k}^{n+1}\right)\right]_{B}\right\|<\varepsilon_{j k}
$$

if $B(j, k) \leq n+1$.

Therefore, relations (1)-(5) are fulfilled for $n+1$. It also follows from (2) and (3) that $\left\{x_{n}\right\}$ and $\left\{w_{j k}\right\}_{k=1}^{\infty}$ are Cauchy sequences for all $j \geq 1$, and from (4) and (5) that the sequences $\left\{b_{j k}\right\}_{k=1}^{\infty}$ are bounded for all $j \geq 1$, where $b_{j k}=b_{j k}^{B(j, k)}$ for all $j, k \geq 1$. Without loss of generality, we may suppose that $\left\{b_{j k}\right\}_{k=1}^{\infty}$ converges weakly to some $b_{j} \in \mathscr{R}_{0}$. Also let $x=\lim x_{n}$ and $w_{j}=\lim _{k} w_{j k}$ for each $j \geq 1$. Then it follows easily that

$$
\left[L_{j}\right]=\left[x \otimes \mathscr{P}_{\mathscr{H}}\left(w_{j}+b_{j}\right)\right]
$$

for all $j \geq 1$.

It follows from (1) that

$$
\begin{aligned}
\|x\| \leq 3 \sum_{j=1}^{\infty} \sum_{k=0}^{\infty} \varepsilon_{j, k}^{1 / 2} & =3 \sum_{j=1}^{\infty} \sum_{k=0}^{\infty} \delta_{j}^{1 / 2}\left(\frac{\theta}{\gamma}\right)^{k / 2} \\
& =\frac{3}{1-(\theta / \gamma)^{1 / 2}} \sum_{j=1} \delta_{j}^{1 / 2} .
\end{aligned}
$$

Similarly, we obtain

$$
\left\|w_{j}\right\| \leq \frac{\delta_{j}^{1 / 2}}{1-\left(\theta(\gamma)^{1 / 2}\right.}
$$

for all $j \geq 1$.

From (4) and (5) we infer that

$$
\begin{aligned}
s_{n+1}\left\|b_{j, k}\right\| & \leq s_{B(j, k-1)+1}\left\|b_{j, k-1}\right\|+\varepsilon_{j, k-1}^{1 / 2} \\
& \leq s_{B(j, 1)+1}\left\|b_{j, 1}\right\|+\sum_{l=1}^{k-1} \varepsilon_{j, l}^{1 / 2} \\
& \leq \sum_{l=0}^{k-1} \varepsilon_{j, l}^{1 / 2}=\sum_{l=0}^{k-1} \delta_{j}^{1 / 2}\left(\frac{\theta}{\gamma}\right)^{l / 2} \leq \delta_{j}^{1 / 2} \frac{1}{1-(\theta / \gamma)^{1 / 2}},
\end{aligned}
$$

and therefore that

$$
\left\|b_{j, k}\right\| \leq \frac{2 \delta_{j}^{1 / 2}}{1-(\theta / \gamma)^{1 / 2}}
$$

for all $j \geq 1$ and $k \geq 0$. 
It follows that

$$
\left\|b_{j}\right\| \leq \frac{2 \delta_{j}^{1 / 2}}{1-(\theta / \gamma)^{1 / 2}} \quad \forall j \geq 1 . \quad \text { Q.E.D. }
$$

From the above relations, we obtain that $T \in \mathbb{A}_{1}, \widehat{\aleph}_{0}(\rho)$,where $\rho \leq \frac{3}{1-(\theta / \gamma)^{1 / 2}}$. The proof is complete.

Added in proof. The complete proofs of the results announced in [2] appear in the paper On the structure of contraction operators. III, Michigan Math. J. 36 (1989), 29-62.

\section{REFERENCES}

1. H. Bercovici, C. Foias, and C. Pearcy, Dual algebras with applications to invariant subspaces and dilation theory, CBMS Regional Conf. Ser. in Math., No. 56, Amer. Math. Soc., Providence, 1985.

2. B. Chevreau, G. Exner, and C. Pearcy, Sur la réflexivité des contractions de l'espace hilbertien, C. R. Acad. Sci. Paris Sér. 1305 (1987), 117-120.

3. B. Chevreau and C. Pearcy, On the structure of contraction operators, J. Funct. Anal. 76 (1988), 1-29.

4. R. Olin and J. Thomson, Algebras of subnormal operators, J. Funct. Anal. 37 (1980), 271301.

Department of Mathematics, InCRest, Bd. Păcil 220, 79622, Bucharest, Romania 